Pacific Journal of Mathematics

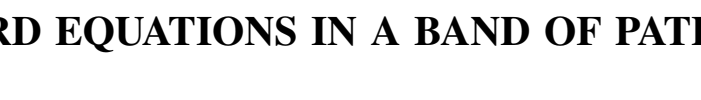




\title{
WORD EQUATIONS IN A BAND OF PATHS
}

\author{
James Nelson, JR. and Mohan S. Putcha
}

In this paper we introduce a multiplication of paths which yields an idempotent semigroup. We study the properties of this band and solve all word equations in this band.

Multiplying paths in a topological space by concatenation is a classical idea in algebraic topology. However, in $\boldsymbol{R}^{n}$, identifying up to homotopy trivializes all paths. There are many ways of obtaining associativity with less identification. Looking only at the images of the paths in $\boldsymbol{R}^{n}$, yields an inverse semigroup (cf. [6]). Lesser identifications lead to semigroups which locally resemble free semigroups $[4,5]$.

1. Preliminaries. Throughout this paper, $\boldsymbol{R}_{0}, \boldsymbol{R}, Z^{+}$will denote the sets of nonnegative reals, reals and positive integers, respectively. If $S$ is a semigroup, then $S^{1}=S$ if $S$ has an identity element; $S^{1}=S \cup\{1\}$ with obvious multiplication if $S$ does not have an identity element. For basic notions of semigroups, see [1].

We will let $\mathscr{Y}$ denote the set of all strictly increasing continuous self-maps $\phi$ of $[0,1]$ with $\phi(0)=0$ and $\phi(1)=1$. Let $n \in Z^{+}$ remain fixed throughout this paper and let $\mathscr{C}$ denote the set of all rectifiable, continuous functions $f$ from $[0,1]$ into $\boldsymbol{R}^{n}$ such that $f(0)=0$ and $f$ is not constant on any subinterval of $[0,1]$. If $f \in \mathscr{M}$, then let $l(f)$ denote the length of $f$. If $f, g \in \mathscr{M}$, then let $f * g \in \mathscr{M}$ be defined by

$$
f * g(x)= \begin{cases}f(2 x) & , 0 \leqq x \leqq 1 / 2 \\ f(1)+g(2 x-1), & 1 / 2 \leqq x \leqq 1\end{cases}
$$

For $f, g \in \mathscr{L}$, define $f \equiv g$ if $g=f \circ \phi$ for some $\phi \in \mathscr{Y}$. Note that if $f \equiv g$, then $l(f)=l(g)$. Intuitively, considered as a function of time, we are interested in the way our path is traced but not the speed. $\equiv$ is an equivalence relation on $\mathscr{L}$. Let $\overline{\mathscr{C}}=\mathscr{M} \mid \equiv$. The operation * defined above remains well defined on $\overline{\mathscr{C}}$ and $\left(\overline{\mathscr{C}}^{*}\right)$ is a cancellative semigroup [4]. (Note that in [4],* was not used to denote this operation.) If $f \in \mathscr{C}$, then let $\bar{f}$ denote the equivalence class of $f$ and define $l(\bar{f})=l(f)$. Then $l(\bar{f})$ is well defined. Let $f \in \mathscr{M}$. For $\alpha \in(0,1]$, define $g(\alpha)=l\left(f_{[0, \alpha]}\right)$ where $f_{[0, \alpha]}$ denotes the path from 0 to $\alpha$ (cf. [4]); $g(0)=0$. By the usual arguments of analysis, $g$ is continuous. So for any $\beta \in(0, l(f))$, there exists $\alpha \in$ 
$(0,1]$ such that $l\left(f_{[0, \alpha]}\right)=\beta$. Let $\mathscr{B}=\overline{\mathscr{C}}^{1}$ and set $l(1)=0$. It follows from the above that for any $a \in \mathscr{B}, \alpha \in[0, l(a)]$, there exist $b, c \in \mathscr{B}$ such that $a=b * c, l(b)=\alpha, l(c)=l(a)-\alpha$. Also note that for any $a, b \in \mathscr{B}, l(a * b)=l(a)+l(b)$. Let $a, b, c, d \in \mathscr{B}$ such that $a * b=c * d, l(a)=l(c)$. Then by [4], $a=c$ and $b=d$.

We now define a new operation on $\mathscr{B}$. Let $a, b \in \mathscr{B}$. First assume $l(a) \leqq l(b)$. Let $a=a_{1} * a_{2}, b=b_{1} * b_{2}$ where $l\left(a_{1}\right)=l\left(a_{2}\right)=l\left(b_{1}\right)$. Then define $a b=a_{1} * b_{2}$. Next assume $l(b) \leqq l(a)$. Let $a=a_{1} * a_{2}, b=b_{1} * b_{2}$ where $l\left(a_{2}\right)=l\left(b_{1}\right)=l\left(b_{2}\right)$. Then define $a b=a_{1} * b_{2}$. From now on when we talk about $\mathscr{B}$, it is to be understood that we are talking about $\mathscr{B}$ with respect to the operation just defined. Visually we can think of the paths colliding and exactly half of the smaller path and an equal part of the larger path destroying each other. Applications, outside of mathematics, of this and similar models will be developed at a later date.

THEOREM 1.1. $\mathscr{B}$ is a band (idempotent semigroup) with identity 1. For any $a, b \in \mathscr{B}, l(a b)=\max .\{l(a), l(b)\}$. For any $a \in \mathscr{B}$, $\mathscr{B}_{a}=\{b \mid b \in \mathscr{B}, l(a)=l(b)\}$ is the rectangular band component of $a$. $\boldsymbol{R}_{0}$, with reversed order, is the maximal semilattice image of $\mathscr{B}$ and $l$ is the corresponding homomorphism.

Proof. First we show that $\mathscr{B}$ is associative. Let $a, b, c \in \mathscr{B}$. We will show $(a b) c=a(b c)$. First assume $l(a) \leqq l(b) \leqq l(c)$. There exist $a_{1}, a_{2}, b_{1}, b_{2}, b_{3}, c_{1}, c_{2}, c_{3} \in \mathscr{B}$ such that $a=a_{1} * a_{2}, b=b_{1} * b_{2} * b_{3}$, $c=c_{1} * c_{2} * c_{3}, l\left(a_{1}\right)=l\left(a_{2}\right)=l\left(b_{1}\right)=l\left(c_{1}\right), l\left(b_{2}\right)=l\left(c_{2}\right)$ and $l\left(b_{1} * b_{2}\right)=l\left(b_{3}\right)$. Then $a b=a_{1} * b_{2} * b_{3}$ and $(a b) c=a_{1} * b_{2} * c_{3}$. Also $b c=b_{1} * b_{2} * c_{3}$ and $a(b c)=a_{1} * b_{2} * c_{3}$. So a $(b c)=(a b) c$. Next assume $l(a) \leqq l(c) \leqq l(b)$. There exist $a_{1}, a_{2}, b_{1}, b_{2}, b_{3}, c_{1}, c_{2} \in \mathscr{B}$ such that $a=a_{1} * a_{2}, b=b_{1} * b_{2} * b_{3}$, $c=c_{1} * c_{2}, l\left(a_{1}\right)=l\left(b_{1}\right)=l\left(a_{2}\right), l\left(b_{3}\right)=l\left(c_{1}\right)=l\left(c_{2}\right)$. Then $a b=a_{1} * b_{2} * b_{3}$ and $(a b) c=a_{1} * b_{2} * c_{2}$. Also $b c=b_{1} * b_{2} * c_{2}$ and a $(b c)=a_{1} * b_{2} * c_{2}$. So $(a b) c=a(b c)$. This takes care of the case when $a$ has smallest length. The case when $c$ has smallestlength is dual. So we are left with the case when $b$ has smallest length. By right-left duality, we can assume $l(b) \leqq l(a) \leqq l(c)$. There exist $a_{1}, a_{2}, a_{3}, b_{1}, b_{2}, c_{1}, c_{2}, c_{3} \in \mathscr{B}$ such that $a=a_{1} * a_{2} * a_{3}, b=b_{1} * b_{2}, c=c_{1} * c_{2} * c_{3}, l\left(a_{1}\right)=l\left(a_{2} * a_{3}\right)=l\left(c_{1} * c_{2}\right)$ and $l\left(b_{1}\right)=l\left(b_{2}\right)=l\left(c_{1}\right)=l\left(a_{3}\right)$. Then $a b=a_{1} * a_{2} * b_{2}$ and $(a b) c=a_{1} * c_{3}$. Also, $b c=b_{1} * c_{2} * c_{3}$ and a $(b c)=a_{1} * c_{3}$. So $(a b) c=a(b c)$ and $\mathscr{B}$ is associative. It is clear that $a^{2}=a$ for all $a \in \mathscr{B}$ and so $\mathscr{B}$ is a band. It is also clear that $l(a b)=\max \{l(a), l(b)\}$ for all $a, b \in \mathscr{B}$. Let $b, c \in \mathscr{B}_{a}$. Then $l(b)=l(c)$. There exist $b_{1}, b_{2}, c_{1}, c_{2} \in \mathscr{B}$ such that $b=b_{1} * b_{2}, c=c_{1} * c_{2}$ and $l\left(b_{1}\right)=l\left(b_{2}\right)=l\left(c_{1}\right)=l\left(c_{2}\right)$. So $b c=b_{1} * c_{2}$ and $b c b=b_{1} * b_{2}=b$. So each $\mathscr{B}_{a}$ is a rectangular band.

Conversely if $R$ is any subrectangular band of $B$ containing $a$ 
and if $b \in R$, then $b a b=b$ so $l(b)=\max \{l(a), l(b)\}$, and hence $l(b) \geqq$ $l(a)$. Similarly $a b a=a$ implies $l(a) \geqq l(b)$, so $l(b)=l(a)$ and $R \leqq B_{a}$.

Lemma 1.2. Let $a, b, c \in \mathscr{B}$ such that $l(b) \leqq l(a)$ and $l(b) \leqq l(c)$. Then $a b c=a c$.

Proof. By symmetry, assume $l(a) \leqq l(c)$. There exist $a_{1}, a_{2}, b_{1}, b_{2}$, $c_{1}, c_{2}, c_{3} \in \mathscr{B}$ such that $a=a_{1} * a_{2}, b=b_{1} * b_{2}, c=c_{1} * c_{2} * c_{3}, \quad l\left(a_{1}\right)=$ $l\left(c_{1} * c_{2}\right)=l\left(a_{2}\right)$ and $l\left(b_{1}\right)=l\left(b_{2}\right)=l\left(c_{1}\right)$. So $b c=b_{1} * c_{2} * c_{3}$ and a $(a b)=$ $a_{1} * c_{3}$. Also, $a c=a_{1} * c_{3}$. So $a(b c)=a c$.

LEMmA 1.3. Let $a, b, c \in \mathscr{B}$ such that $l(a) \leqq l(b) \leqq l(c)$ and $a c=b c$. Then $a b=b$ and there exists $a^{\prime} \in \mathscr{B}$ such that $l(a)=l\left(a^{\prime}\right)$ and $c=a^{\prime} b c$.

Proof. Let $a=a_{1} * a_{2}, b=b_{1} * b_{2} * b_{3}, c=c_{1} * c_{2} * c_{3}$ with $l\left(a_{1}\right)=$ $l\left(a_{2}\right)=l\left(b_{1}\right)=l\left(c_{1}\right), l\left(b_{2}\right)=l\left(c_{2}\right)$ and $l\left(b_{1} * b_{2}\right)=l\left(b_{3}\right)$. Then $a c=a_{1} * c_{2} * c_{3}$ and $b c=b_{1} * b_{2} * c_{3}$. So $a_{1}=b_{1}$ and $b_{2}=c_{2}$. Thus, $a b=a_{1} * b_{2} * b_{3}=$ $b_{1} * b_{2} * b_{3}=b$. Let $a^{\prime}=c_{1} * a_{2}$. Then $l(a)=l\left(a^{\prime}\right)$ and $a^{\prime} b=c_{1} * b_{2} * b_{3}$. So $a^{\prime} b c=c_{1} * b_{2} * c_{3}=c_{1} * c_{2} * c_{3}=c$.

Following is the right-left dual of Lemma 1.3.

LEMMA 1.4. Let $a, b, c \in \mathscr{B}$ such that $l(a) \leqq l(b) \leqq l(c)$ and $c a=c b$. Then $b a=b$ and there exists $a^{\prime} \in \mathscr{B}$ such that $l(a)=l\left(a^{\prime}\right)$ and $c=c b a^{\prime}$.

LEMma 1.5. Let $a, b, c \in \mathscr{B}$ such that $a b c=b$. Then for $d \in \mathscr{B}$, the following are equivalent.

(1) $a d c=b$ and $l(d)=l(b)$.

(2) $d=a^{\prime} b c^{\prime}$ for some $a^{\prime}, c^{\prime} \in \mathscr{B}$ with $l(a)=l\left(a^{\prime}\right)$ and $l(c)=l\left(c^{\prime}\right)$.

Proof. First note that $l(a) \leqq l(b)$ and $l(c) \leqq l(b)$.

$(1) \Rightarrow(2)$. By Lemma 1.2, $a d=a d c d=b d$. Similarly $d c=d b$. By Lemmas 1.3 and 1.4, there exists $a^{\prime}, c^{\prime} \in \mathscr{B}$ such that $l(a)=l\left(a^{\prime}\right)$, $l(c)=l\left(c^{\prime}\right)$ and $d=a^{\prime} b d=d b c^{\prime}$. So $d=a^{\prime} b d b c^{\prime}=a^{\prime} b c^{\prime}$.

$(2) \Rightarrow(1)$. Clearly $l(d)=l(b)$. By Lemma 1.2, $a d c=a a^{\prime} b c^{\prime} c=$ $a b c=b$.

LEMMA 1.6. Let $a, b, c, d \in \mathscr{B}$ such that $l(b) \geqq l(a), \quad l(c) \geqq l(d)$ and $a b c d=b c$. Then $a b=b$ and $c d=c$.

Proof. Since $l(b c) \geqq l(d), a b c=a b c d b c=b c . \quad$ So $(a b) b c=b(b c)$. Since $l(a b)=l(b) \leqq l(b c)$, Lemma 1.3 implies that $(a b) b=b$. So $a b=b$. The other assertion is proved dually using Lemma 1.4 . 
LEMMA 1.7. Let $a_{1}, a_{2}, b_{1}, b_{2}, c \in \mathscr{B}$ such that $l(c) \geqq l\left(a_{1}\right), l\left(a_{2}\right)$, $l\left(b_{1}\right) l\left(b_{2}\right)$ and $a_{1} c a_{2}=b_{1} c b_{2}$. Then

(i) If $l\left(a_{1}\right) \leqq l\left(b_{1}\right)$ and $l\left(b_{2}\right) \leqq l\left(a_{2}\right)$, then $a_{1} b_{1} a_{2} b_{2}=b_{1} a_{2}$.

(ii) If $l\left(a_{1}\right) \leqq l\left(b_{1}\right)$ and $l\left(a_{2}\right) \leqq l\left(b_{2}\right)$, then $a_{1} b_{1} b_{2} a_{2}=b_{1} b_{2}$.

Proof. $a_{1} c a_{2} c=b_{1} c b_{2} c$ and so by Lemma 1.2, $a_{1} c=b_{1} c$. Similarly $c a_{2}=c b_{2}$. $b_{1} a_{2}$.

(i) By Lemmas 1.3 and 1.4, $a_{1} b_{1}=b_{1}$ and $a_{2} b_{2}=a_{2}$. So $a_{1} b_{1} a_{2} b_{2}=$

(ii) By Lemmas 1.3 and 1.4, $a_{1} b_{1}=b_{1}$ and $b_{2} a_{2}=b_{2}$. So $a_{1} b_{1} b_{2} a_{2}=$ $b_{1} b_{2}$.

LEMMA 1.8. Let $a_{1}, a_{2}, b_{1}, b_{2} \in \mathscr{B}$ such that $l\left(b_{1}\right) \geqq l\left(a_{1}\right), l\left(a_{2}\right) \geqq l\left(b_{2}\right)$ and $a_{1} b_{1} a_{2} b_{2}=b_{1} a_{2}$. Then for $c \in \mathscr{B}$, the following are equivalent.

(1) $a_{1} c a_{2}=b_{1} c b_{2}, l(c) \geqq l\left(b_{1}\right)$ and $l(c) \geqq l\left(a_{2}\right)$.

(2) $c=a_{1}^{\prime} b_{1} d a_{2} b_{2}^{\prime}$ for some $a_{1}^{\prime}, b_{2}^{\prime}, d \in \mathscr{B}$ with $l\left(a_{1}\right)=l\left(a_{1}^{\prime}\right), l\left(b_{2}\right)=$ $l\left(b_{2}^{\prime}\right), l(d) \geqq l\left(b_{1}\right), l(d) \geqq l\left(a_{2}\right)$.

Proof. (1) $\Rightarrow(2) . \quad a_{1} c a_{2} c=b_{1} c b_{2} c$ and so $a_{1} c=b_{1} c$. Similarly $c a_{2}=c b_{2}$. By Lemmas 1.3 and 1.4, there exist $a_{1}^{\prime}, b_{2}^{\prime} \in \mathscr{B}$ such that $l\left(a_{1}\right)=l\left(a_{1}^{\prime}\right), l\left(b_{2}\right)=l\left(b_{2}^{\prime}\right)$ and $c=a_{1}^{\prime} b_{1} c=c a_{2} b_{2}^{\prime}$. So $c=a_{1}^{\prime} b_{1} c a_{2} b_{1}^{\prime}$.

$(2) \Rightarrow(1)$. By Lemma 1.6, $a_{1} b_{1}=b_{1}$ and $a_{2} b_{2}=a_{2}$. By Lemma 1.2, $a_{1} c a_{2}=a_{1} a_{1}^{\prime} b_{1} d a_{2} b_{2}^{\prime} a_{2}=a_{1} b_{1} d a_{2}=b_{1} d a_{2}$. Also $b_{1} c b_{2}=b_{1} a_{1}^{\prime} b_{1} d a_{2} b_{2}^{\prime} b_{2}=b_{1} d a_{2} b_{2}=$ $b_{1} d a_{2}$. So $a_{1} c a_{2}=b_{1} c b_{2} \quad$ Clearly $l(c) \geqq l\left(b_{1}\right), l(c) \geqq l\left(a_{2}\right)$.

LEMMA 1.9. Let $a_{1}, a_{2}, b_{1}, b_{2} \in \mathscr{B}$ such that $l\left(b_{1}\right) \geqq l\left(a_{1}\right), l\left(b_{2}\right) \geqq$ $l\left(a_{2}\right)$ and $a_{1} b_{1} b_{2} a_{2}=b_{1} b_{2}$. Then for $c \in \mathscr{B}$, the following are equivalent.

(1) $a_{1} c a_{2}=b_{1} c b_{2}$ and $l(c) \geqq l\left(b_{1}\right), l(c) \geqq l\left(b_{2}\right)$.

(2) $c=a_{1}^{\prime} b_{1} d b_{2} a_{2}^{\prime}$ for some $d, a_{1}^{\prime}, a_{2}^{\prime} \in \mathscr{B}$ with $l\left(a_{1}\right)=l\left(a_{1}^{\prime}\right), l\left(a_{2}\right)=$ $l\left(a_{2}^{\prime}\right), l(d) \geqq l\left(b_{1}\right)$ and $l(d) \geqq l\left(b_{2}\right)$.

Proof. (1) $\Rightarrow(2) . \quad a_{1} c a_{2} c=b_{1} c b_{2} c$ and so $a_{1} c=b_{1} c$. Similarly $c a_{2}=c b_{2}$. By Lemmas 1.3 and 1.4, there exist $a_{1}^{\prime}$, $a_{2}^{\prime}$ such that $c=a_{1}^{\prime} b_{1} c=c b_{2} a_{2}^{\prime}, l\left(a_{1}\right)=l\left(a_{1}^{\prime}\right)$ and $l\left(a_{2}\right)=l\left(a_{2}^{\prime}\right)$. Then $c=a_{1}^{\prime} b_{1} c b_{2} a_{2}^{\prime}$.

$(2) \Rightarrow(1)$. By Lemma 1.6, $a_{1} b_{1}=b_{1}$ and $b_{2} a_{2}=b_{2}$. Then by Lemma 1.2, $a_{1} c a_{2}=a_{1} a_{1}^{\prime} b_{1} d b_{2} a_{2}^{\prime} a_{2}=a_{1} b_{1} d b_{2} a_{2}=b_{1} d b_{2}$. Also $b_{1} c b_{2}=$ $b_{1} a_{1}^{\prime} b_{1} d b_{2} a_{2}^{\prime} b_{2}=b_{1} d b_{2}$. So $a_{1} c a_{2}=b_{1} c b_{2}$. Clearly, $l(c) \geqq l\left(b_{1}\right)$ and $l(c) \geqq$ $l\left(b_{2}\right)$.

2. Word equations. If $\Gamma$ is a nonempty set, then let $\mathscr{F}=$ $\mathscr{F}(\Gamma)$ denote the free semigroup on $\Gamma$. If $\Gamma=\left\{X_{1}, \cdots, X_{m}\right\}, w=$ $w\left(X_{1}, \cdots, X_{m}\right) \in \mathscr{F}^{1}$ and $a_{1}, \cdots, a_{m} \in \mathscr{B}$, then let $w\left(a_{1}, \cdots, a_{m}\right)$ be the element of $\mathscr{B}$ obtained by replacing $X_{1}, \cdots, X_{m}$ in $w$ by $a_{1}, \cdots, a_{m}$ 
respectively; if $w=1$, then $w\left(a_{1}, \cdots, a_{m}\right)=1$. For introduction to word equations in free semigroups, see $[2,3]$.

Definition. By a word equation in variables $X_{1}, \cdots, X_{m}$ we mean $\left\{w_{1}, w_{2}\right\}$ where $w_{1}=w_{1}\left(X_{1}, \cdots, X_{m}\right), \quad w_{2}=w_{2}\left(X_{1}, \cdots, X_{m}\right) \epsilon$ $\mathscr{F}\left(X_{1}, \cdots, X_{m}\right)^{1}$. It is not necessary that each $X_{i}$ appears in $w_{1} w_{2}$. By a solution of $\left\{w_{1}, w_{2}\right\}$ in $\mathscr{B}$, we mean $\left(a_{1}, \cdots, a_{m}\right)$ where $a_{1}, \cdots, a_{m} \in \mathscr{B}$ and $w_{1}\left(a_{1}, \cdots, a_{m}\right)=w_{2}\left(a_{1}, \cdots, a_{m}\right)$. A solution $\left(a_{1}, \cdots, a_{m}\right)$ is an ordered solution if $l\left(a_{1}\right) \leqq l\left(a_{2}\right) \leqq \cdots \leqq l\left(a_{m}\right)$.

REMARK 2.1. In the above situation, note that the solutions of $\left\{w_{1}, w_{2}\right\}$ are exactly all the ordered solutions obtained by relabeling the $X_{i}$ 's in the $m$ ! possible ways. So we will concentrate on obtaining the ordered solutions of word equations.

TheOREM 2.2. Let $m \in Z^{+}, m \geqq 2$. Let $w_{1}, w_{2} \in \mathscr{F}=\mathscr{F}\left(X_{1}, \cdots, X_{m}\right)$. Suppose $w_{1}=u_{1} X_{m} u_{2}$ for some $u_{1}, u_{2} \in \mathscr{F}^{1}$ such that $X_{m}$ does not occur in $u_{1}, u_{2}, w_{2}$, and $X_{m-1}$ occurs in $w_{1} w_{2}$. Let $v_{1}=u_{1} w_{2} u_{2}$. Consider the word quation $\left\{v_{1}, v_{2}\right\}$ in variables $X_{1}, \cdots, X_{m-1}$. Let $\left(a_{1}, \cdots, a_{m-1}\right)$ be an ordered solution of $\left\{v_{1}, w_{2}\right\}$ in $\mathscr{B}$. Set $a=$ $u_{1}\left(a_{1}, \cdots, a_{m-1}\right), b=w_{2}\left(a_{1}, \cdots, a_{m-1}\right), c=u_{2}\left(a_{1}, \cdots, a_{m-1}\right)$. Let $a^{\prime}, c^{\prime} \in \mathscr{B}$ such that $l(a)=l\left(a^{\prime}\right)$ and $l(c)=l\left(c^{\prime}\right)$. If $a_{m}=a^{\prime} b c^{\prime}$, then $\left(a_{1}, \cdots\right.$, $\left.a_{m-1}, a_{m}\right)$ is an ordered solution of $\left\{w_{1}, w_{2}\right\}$. Moreover, every ordered solution of $\left\{w_{1}, w_{2}\right\}$ in $\mathscr{B}$ is obtained in this manner.

Proof. Let $\left(a_{1}, \cdots, a_{m}\right)$ be an ordered solution of $\left\{w_{1}, w_{2}\right\}$. Let $a=u_{1}\left(a_{1}, \cdots, a_{m-1}\right), \quad b=w_{2}\left(a_{1}, \cdots, a_{m-1}\right), \quad c=u_{2}\left(a_{1}, \cdots, a_{m-1}\right)$. Then $a a_{m} c=b$. Clearly then, $a b c=a a a_{m} c c=a a_{m} c=b$. So $\left(a_{1}, \cdots, a_{m-1}\right)$ is an ordered solution of $\left\{v_{1}, w_{2}\right\}$. New it follows from Lemma 1.5, that $a_{m}$ has the prescribed form. The converse also follows from Lemma 1.5 .

In what follows, if $w \in \mathscr{F}\left(X_{1}, \cdots, X_{m}\right)^{1}$, then let $\theta(w)=$ $\max \left\{i \mid X_{i}\right.$ appears in $\left.w\right\} ; \theta(1)=0$.

THEOREM 2.3. Let $m \in Z^{+}, m \geqq 2$. Let $w_{1}, w_{2} \in \mathscr{F}=\mathscr{F}\left(X_{1}, \cdots, X_{m}\right)$. Suppose $w_{1}=u_{1} X_{m} u_{2}, w_{2}=v_{1} X_{m} v_{2}$ for some $u_{1}, u_{2}, v_{1}, v_{2} \in \mathscr{F}^{1}$ such that $X_{m}$ does not appear in $u_{1} u_{2} v_{1} v_{2}$.

(i) Suppose $\theta\left(u_{1}\right) \leqq \theta\left(v_{1}\right)$ and $\theta\left(v_{2}\right) \leqq \theta\left(u_{9}\right)$. Let $f_{1}=u_{1} v_{1} u_{2} v_{2}$ and $f_{2}=v_{1} u_{2}$. Consider the word equation $\left\{f_{1}, f_{2}\right\}$ in variables $X_{1}, \cdots, X_{m-1}$ and let $\left(a_{1}, \cdots, a_{m-1}\right)$ be an ordered solution of $\left\{f_{1}, f_{2}\right\}$ in $\mathscr{B}$. Set $A_{1}=u_{1}\left(a_{1}, \cdots, a_{m-1}\right), \quad A_{2}=u_{2}\left(a_{1}, \cdots, a_{m-1}\right), \quad B_{1}=v_{1}\left(a_{1}, \cdots, a_{m-1}\right)$ and $B_{2}=v_{2}\left(a_{1}, \cdots, a_{m-1}\right)$. Let $A_{1}^{\prime}, B_{2}^{\prime}, D \in \mathscr{B}$ such that $l\left(A_{1}^{\prime}\right)=l\left(A_{1}\right), l\left(B_{2}^{\prime}\right)=$ $l\left(B_{2}\right), l(D) \geqq l\left(a_{m-1}\right)$. Set $a_{m}=A_{1}^{\prime} B_{1} D A_{2} B_{2}^{\prime}$. Then $\left(a_{1}, \cdots, a_{m-1}, a_{m}\right)$ is an ordered solution of $\left\{w_{1}, w_{2}\right\}$. Moreover, every ordered solution of 
$\left\{w_{1}, w_{2}\right\}$ in $\mathscr{B}$ is obtained in this manner.

(ii) Suppose $\theta\left(u_{1}\right) \leqq \theta\left(v_{1}\right)$ and $\theta\left(u_{2}\right) \leqq \theta\left(v_{2}\right)$. Let $f_{1}=u_{1} v_{1} v_{2} u_{2}$, $f_{2}=v_{1} v_{2}$. Consider the word equation $\left\{f_{1}, f_{2}\right\}$ in variables $X_{1}, \cdots, X_{m-1}$. Let $\left(a_{1}, \cdots, a_{m-1}\right)$ be an ordered solution of $\left\{f_{1}, f_{2}\right\}$ in $\mathscr{B}$. Set $A_{1}=$ $u_{1}\left(a_{1}, \cdots, a_{m-1}\right), A_{2}=u_{2}\left(a_{1}, \cdots, a_{m-1}\right), B_{1}=v_{1}\left(a_{1}, \cdots, a_{m-1}\right)$ and $B_{2}=$ $v_{2}\left(a_{1}, \cdots, a_{m-1}\right)$. Let $A_{1}^{\prime}, A_{2}^{\prime}, D \in \mathscr{B}$ such that $l\left(A_{1}^{\prime}\right)=l\left(A_{1}\right), l\left(A_{2}^{\prime}\right)=l\left(A_{2}\right)$, $l(D) \geqq l\left(a_{m-1}\right)$. Set $a_{m}=A_{1}^{\prime} B_{1} D B_{2} A_{2}^{\prime}$. Then $\left(a_{1}, \cdots, a_{m-1}, a_{m}\right)$ is an ordered solution of $\left\{w_{1}, w_{2}\right\}$. Moreover, every ordered solution of $\left\{w_{1}, w_{2}\right\}$ in $\mathscr{B}$ is obtained in this manner.

Proof. Suppose $\left(a_{1}, \cdots, a_{m}\right)$ is an ordered solution of $\left\{w_{1}, w_{2}\right\}$ in $\mathscr{B}$. Let $A_{1}=u_{1}\left(a_{1}, \cdots, a_{m-1}\right), A_{2}=u_{2}\left(a_{1}, \cdots, a_{m-1}\right), B_{1}=v_{1}\left(a_{1}, \cdots, a_{m-1}\right)$ and $B_{2}=v_{2}\left(a_{1}, \cdots, a_{m-1}\right)$. So $A_{1} a_{m} A_{2}=B_{1} a_{m} B_{2}$.

(i) We have $l\left(A_{1}\right) \leqq l\left(B_{1}\right) \leqq l\left(a_{m}\right), \quad l\left(B_{2}\right) \leqq l\left(A_{2}\right) \leqq l\left(a_{m}\right) . \quad$ By Lemma 1.7(i), $A_{1} B_{1} A_{2} B_{2}=B_{1} A_{2}$. So $\left(a_{1}, \cdots, a_{m-1}\right)$ is an ordered solution of $\left\{f_{1}, f_{2}\right\}$. That $a_{m}$ has the required form, follows from Lemma 1.8. The converse also follows from Lemma 1.8.

(ii) We have $l\left(A_{1}\right) \leqq l\left(B_{1}\right) \leqq l\left(a_{m}\right), \quad l\left(A_{2}\right) \leqq l\left(B_{2}\right) \leqq l\left(a_{m}\right) . \quad$ By Lemma 1.7(ii), $A_{1} B_{1} B_{2} A_{2}=B_{1} B_{2}$. So $\left(a_{1}, \cdots, a_{m-1}\right)$ is an ordered solution of $\left\{f_{1}, f_{2}\right\}$. That $a_{m}$ has the required form, follows from Lemma 1.9. The converse also follows from Lemma 1.9 .

THEOREM 2.4. Let $m \in Z^{+}, m \geqq 2, w_{1}, w_{2} \in \mathscr{F}=\mathscr{F}\left(X_{1}, \cdots, X_{m}\right)$.

(i) Suppose $w_{1}=u_{1} X_{m} u_{2} X_{m} u_{3}$ for some $u_{1}, u_{2}, u_{3} \in \mathscr{F}^{1}$ such that $X_{m}$ does not occur in $u_{1} u_{3} w_{2}$. Let $f_{1}=u_{1} X_{m} u_{3}, f_{2}=w_{2}$. Then the ordered solutions of $\left\{w_{1}, w_{2}\right\}$ in $\mathscr{B}$ are exactly the same as the ordered solutions of $\left\{f_{1}, f_{2}\right\}$.

(ii) Suppose $w_{1}=u_{1} X_{m} u_{2} X_{m} u_{3}, \quad w_{2}=v_{1} X_{m} v_{2} X_{m} v_{3}$ for some $u_{1}, u_{2}, u_{3}, v_{1}, v_{2}, v_{3} \in \mathscr{F}^{1}$ such that $X_{m}$ does not occur in $u_{1} u_{3} v_{1} v_{3}$. Let $f_{1}=u_{1} X_{m} u_{3}, f_{2}=v_{1} X_{m} v_{3}$. Then the ordered solutions of $\left\{w_{1}, w_{2}\right\}$ in $\mathscr{B}$ are exactly the same as the ordered solutions of $\left\{f_{1}, f_{2}\right\}$.

(iii) Suppose $w_{1}=u_{1} X_{m} u_{2} X_{m} u_{3}, w_{2}=v_{1} X_{m} v_{2}$ for some $u_{1}, u_{2}, u_{3}$, $v_{1}, v_{2} \in \mathscr{F}^{1}$ such that $X_{m}$ does not occur in $u_{1} u_{3} v_{1} v_{2}$. Let $f_{1}=u_{1} X_{m} u_{3}$, $f_{2}=v_{1} X_{m} v_{2}$. Then the ordered solutions of $\left\{w_{1}, w_{2}\right\}$ in $\mathscr{B}$ are exactly the same as the ordered solutions of $\left\{f_{1}, f_{2}\right\}$.

Proof. Let $a_{1}, \cdots, a_{m} \in \mathscr{B}$ such that $l\left(a_{1}\right) \leqq \cdots \leqq l\left(a_{m}\right)$. Then in all cases $f_{1}\left(a_{1}, \cdots, a_{m}\right)=w_{1}\left(a_{1}, \cdots, a_{m}\right)$ and $f_{2}\left(a_{1}, \cdots, a_{m}\right)=$ $w_{2}\left(a_{1}, \cdots, a_{m}\right)$.

REMARK 2.5. Let $\left\{w_{1}, w_{2}\right\}$ be a word equation in variables $X_{1}, \cdots, X_{m}$. If $w_{1}$ or $w_{2}=1$, the solutions are obvious. So assume $w_{1} \neq 1, w_{2} \neq 1$. If $m=1$, the solutions are again obvious. So let $m \geqq 2$. We claim that the ordered solutions of $\left\{w_{1}, w_{2}\right\}$ can be 
described in terms of ordered solutions of a certain word equation in $m-1$ variables. If some $X_{j}$ does not appear in $w_{1} w_{2}$, then $\left\{w_{1}, w_{2}\right\}$ can itself be considered as a word equation in $m-1$ variables and our claim holds trivially. Otherwise either $\left\{w_{1}, w_{2}\right\}$ or $\left\{w_{2}, w_{1}\right\}$ satisfies Theorems 2.2, 2.3 or 2.4 and our claim still holds. Thus given any word equation, we can completely describe all its solutions in $\mathscr{B}$.

ExAMPLE 2.6. Consider the word equation $\{B C B A, C A B A C\}$ in variables $A, B, C$. There are six ways of ordering $A, B, C$. Finding the ordered solutions for all these equations (using the theorems of this section) and simplifying, we see that following is the list of all solutions in $\mathscr{B}$ of the above word equation.

$$
\begin{aligned}
& A=a \\
& B=b \\
& C=b c b a,
\end{aligned}
$$

where $a, b, c \in \mathscr{B}$.

$$
\begin{aligned}
& A=c^{\prime} b a c \\
& B=c b \\
& C=c
\end{aligned}
$$

where $a, b, c, c^{\prime} \in \mathscr{B}, l(c)=l\left(c^{\prime}\right) \leqq l(b) \leqq l(a)$.

$$
\begin{aligned}
& A=c^{\prime} b a b c \\
& B=b \\
& C=b c
\end{aligned}
$$

where $a, b, c, c^{\prime} \in \mathscr{B}, l(b) \leqq l(c)=l\left(c^{\prime}\right) \leqq l(a)$.

$$
\begin{aligned}
& A=a c \\
& B=c a b a^{\prime} \\
& C=c
\end{aligned}
$$

where $a, a^{\prime}, b, c \in \mathscr{B}, l(c) \leqq l(a)=l\left(a^{\prime}\right) \leqq l(b)$.

$$
\begin{aligned}
& A=a \\
& B=c b c a^{\prime} \\
& C=c a
\end{aligned}
$$

where $a, a^{\prime}, b, c \in \mathscr{B}, l(a)=l\left(a^{\prime}\right) \leqq l(c) \leqq l(b)$.

3. Concluding remarks. Instead of starting with the semigroup of paths, we can start with semigroup of designs around the 
unit disc of [5] and analogously define a new idempotent, associative multiplication. Then the results of this paper remain true for that band. More generally, let $E$ be any band with an identity element. Let $\Omega$ be its maximal semilattice image and $l$ the corresponding homomorphism. Consider $\Omega$ with the order given by $e \leqq f$ if and only if $e f=f$. If $\Omega$ is linearly ordered and if Lemmas 1.2, 1.3 and 1.4 are true for $\mathscr{E}$, then all of $\S 2$ remains true for $\mathscr{E}$.

\section{REFERENCES}

1. A. H. Clifford and G. B. Preston, The algebraic theory of semigroups, vol. 1, Amer. Math. Soc., Providence, Rhode Island, 1961.

2. Ju. I. Hmelevskii, Equations in the free semigroup, Trudy Matem. Inst. im. Steklova, 107 (1971), 288 pp. (Russian).

3. A. Lentin, Équations dans les monoides libres, Mouton/Gauthier-Villars, 1972.

4. M. S. Putcha, Word equations of paths, J. of Algebra, (to appear).

5. - Word equations in some geometric semigroups, Pacific J. Math., 75 (1978), 243-266.

6. M. S. Putcha and A. H. Schoenfeld, Applications of algebraic and combinatoric techniques to a problem in geometry, J. of Pure and Applied Algebra, 7 (1976), 235-237.

Received March 21, 1977 and in revised form February 8, 1978. The first author was partially supported by Engineering Foundation, North Carolina State University, and the second author was partially supported by NSF Grant MCS 76-05784.

North Carolina State University

RALEIGH, NC 27650 


\section{PACIFIC JOURNAL OF MATHEMATICS}

\section{EDITORS}

DoNALD BABBITT (Managing Editor)

University of California

Los Angeles, California 90024

HUGo Rossi

University of Utah

Salt Lake City, UT 84112

C. C. MOORE and ANDREW OGG

University of California

Berkeley, CA 94720
J. DUGUNDJI

Department of Mathematics University of Southern Californı Los Angeles, California 90007

R. Finn and J. Milgram Stanford University Stanford, California 94305

\section{ASSOCIATE EDITORS}

E. F. BECKENBACH

B. H. Neumann
F. WOLF

K. YOSHIDA

\section{SUPPORTING INSTITUTIONS}

UNIVERSITY OF BRITISH COLUMBIA CALIFORNIA INSTITUTE OF TECHNOLOGY UNIVERSITY OF CALIFORNIA MONTANA STATE UNIVERSITY UNIVERSITY OF NEVADA, RENO NEW MEXICO STATE UNIVERSITY OREGON STATE UNIVERSITY UNIVERSITY OF OREGON
UNIVERSITY OF SOUTHERN CALIFORNIA STANFORD UNIVERSITY UNIVERSITY OF HAWAII UNIVERSITY OF TOKYO UNIVERSITY OF UTAH WASHINGTON STATE UNIVERSITY UNIVERSITY OF WASHINGTON 


\section{Pacific Journal of Mathematics}

Vol. 82, No. $1 \quad$ January, 1979

Werner Bäni, Subspaces of positive definite inner product spaces of countable dimension ...................................... 1

Marilyn Breen, The dimension of the kernel of a planar set..............

Kenneth Alfred Byrd, Right self-injective rings whose essential right ideals

are two-sided

Patrick Cousot and Radhia Cousot, Constructive versions of Tarski's fixed

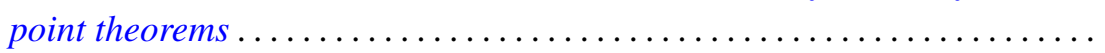

Ralph S. Freese, William A. Lampe and Walter Fuller Taylor, Congruence lattices of algebras of fixed similarity type. $I \ldots \ldots \ldots \ldots \ldots \ldots \ldots$

Cameron Gordon and Richard A. Litherland, On a theorem of Murasugi .....

Mauricio A. Gutiérrez, Concordance and homotopy. I. Fundamental

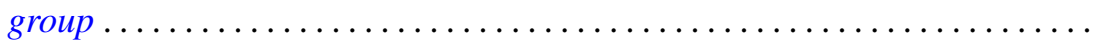

Richard I. Hartley, Metabelian representations of knot groups .............

Ted Hurley, Intersections of terms of polycentral series of free groups and free

Lie algebras ........................................

Roy Andrew Johnson, Some relationships between measures ............ 117

Oldřich Kowalski, On unitary automorphisms of solvable Lie algebras .......

Kee Yuen Lam, $K O$-equivalences and existence of nonsingular bilinear

maps...................................................

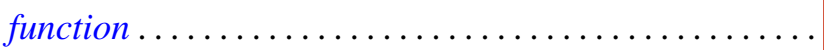

Robert A. Messer and Alden H. Wright, Embedding open 3-manifolds in compact 3-manifolds ............................

Gerald Ira Myerson, A combinatorial problem in finite fields. I . .

James Nelson, Jr. and Mohan S. Putcha, Word equations in a band of paths.

Baburao Govindrao Pachpatte and S. M. Singare, Discrete generalized Gronwall inequalities in three independent variables . .

William Lindall Paschke and Norberto Salinas, $C^{*}$-algebras associated with free products of groups ........................

Bruce Reznick, Banach spaces with polynomial norms ....

David Rusin, What is the probability that two elements of a finite group

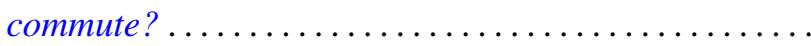

M. Shafii-Mousavi and Zbigniew Zielezny, On hypoelliptic differential operators of constant strength ...

Joseph Gail Stampfli, On selfadjoint derivation ranges .... . . .

Robert Charles Thompson, The case of equality in the matrix-valued triangle

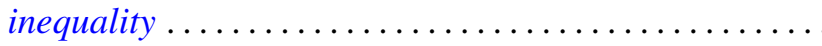

Marie Angela Vitulli, The obstruction of the formal moduli space in the negatively graded case. 